\title{
Novel quinolines carrying pyridine, thienopyridine, isoquino- line, thiazolidine, thiazole and thiophene moieties as potential anticancer agents
}

\author{
MOSTAFA M. GHORAB ${ }^{1 *}$ \\ MANSOUR S. ALSAID ${ }^{1}$ \\ MOHAMMED S. AL-DOSARI ${ }^{1}$ \\ FATMA A. RAGAB ${ }^{2}$ \\ ABDULLAH A. AL-MISHARI ${ }^{3}$ \\ ABDULAZIZ N. ALMOQBIL ${ }^{3}$ \\ ${ }^{1}$ Department of Pharmacognosy \\ College of Pharmacy \\ King Saud University \\ P.O. Box 2457, Riyadh 11451 \\ Kingdom of Saudi Arabia \\ ${ }^{2}$ Pharmaceutical Chemistry Department \\ Faculty of Pharmacy \\ Cairo University, Cairo 11562, Egypt \\ ${ }^{3}$ Medicinal, Aromatic and Poisonous \\ Plants Research Center (MAPPRC) \\ College of Pharmacy \\ King Saud University, P.O. Box 2457 \\ Riyadh 11451, Saudi Arabia
}

\begin{abstract}
As a part of ongoing studies in developing new anticancer agents, novel 1,2-dihydropyridine 4, thienopyridine 5, isoquinolines 6-20, acrylamide 21, thiazolidine 22, thiazoles 23-29 and thiophenes 33-35 bearing a biologically active quinoline nucleus were synthesized. The structure of newly synthesized compounds was confirmed on the basis of elemental analyses and spectral data. All the newly synthesized compounds were evaluated for their cytotoxic activity against the breast cancer cell line MCF7. 2,3-Dihydrothiazole-5-carboxamides 27, 25, 4,5,6,7-tetrahydrobenzo[b]thiophene-3carboxamide (34), 1,2-dihydroisoquinoline-7-carbonitrile (7), 5,6,7,8-tetrahydro-4H-cyclohepta[b]thiophene-3-carboxamide (35), 1,2-dihydroisoquinoline-7-carbonitrile (6), 2-cyano-3-(dimethylamino)- $N$-(quinolin-3-yl)acrylamide (21), 1,2-dihydroisoquinoline-7-carbonitriles (11) and (8) exhibited higher activity $\left(I C_{50}\right.$ values of $\left.27-45 \mu \mathrm{mol} \mathrm{L}^{-1}\right)$ compared to doxorubicin $\left(I C_{50} 47.9 \mu \mathrm{mol} \mathrm{L}{ }^{-1}\right)$. LQ quinolin-3-yl)-1,2-dihydroisoquinoline-7-carbonitrile (12), 2-thioxo-2,3-dihydrothiazole-5-carboxamide (28) and quinolin-3-yl)-1,2-dihydroisoquinoline-7-carbonitrile (15) show activity comparable to doxorubicin, while (quinolin-3-yl)-1,2-dihydroisoquinoline7-carbonitrile (9), 2,3-dihydrothiazole-5-carboxamide (24), thieno $[3,4-c]$ pyridine-4(5H)-one (5), cyclopenta[b]thiophene-3-carboxamide (33) and (quinolin-3-yl)-6-stryl-1,2dihydroisoquinoline-7-carbonitrile (10) exhibited moderate activity, lower than doxorubicin.
\end{abstract}

Keywords: quinolines, pyridine, thienopyridine, isoquinoline, acrylamide, thiazole, thiophene, anticancer activity

Cancer is one of the most critical health issues and is considered the second leading cause of death worldwide, just after circulatory diseases. Despite the availability of improved drugs including targeted cancer therapies, the worldwide cancer burden is expected to increase by as much as 15 million new cancer cases per year by 2020, according

\footnotetext{
*Correspondence; e-mail: mmsghorab@yahoo.com
} 
to the World Health Organization (WHO), unless further preventive measures are put into practice (1). Quinoline scaffold plays an important role in anticancer drug development since their derivatives have shown excellent results through different mechanisms of action such as growth inhibition by cell cycle arrest, apoptosis, inhibition of angiogenesis, disruption of cell migration and modulation of nuclear receptor responsiveness. The anticancer potential of several of these derivatives has been demonstrated on various cancer cell lines. In addition, many of quinoline derivatives are useful in diverse applications including pharmaceuticals and are today available as drugs (2). Therefore there is an urgent need to develop new classes of chemotherapeutic agents with different mechanisms of action to treat cancer (3). It has been reported that designing a single molecule with more than one pharmacophore with different modes of action could be beneficial for the treatment of cancer (4), as well as for reducing unwanted side effects (5). Many quinolines, dihydropyridines, thienopyridines, isoquinolines, acrylamides, thiazolidines, thiazoles and thiophenes were found to possess antineoplastic activity (6-11). Although the antineoplastic activity of these quinolines was attributed to intercalating binding to DNA, there were additional advantages of quinolines that interact with DNA, with a low association constant. The corresponding significant increase in the amount of free drug at equilibrium may have an important effect upon the distribution and hence the spectrum of activity and accessibility of these molecules to solid tumors $(12,13)$. On the other hand, a number of quinoline derivatives were reported to reverse tumor cell multidrug resistance $(14,15)$. Recently, it has been demonstrated that $10 \mu \mathrm{mol} \mathrm{L}^{-1}$ chloroquine significantly increases cancer cell killing effects $(4,16)$. Several chloroquinolines (CQ) known as antimalarial drugs, such as $N^{1}$-(7-chloroquinolin-4-yl)- $N^{2}, N^{2}$-diethylethane-1,2-diamine (I), $N^{1}, N^{1}$-diethyl- $N^{2}$-(7-fluoroquinolin-4-yl)ethane-1,2-diamine (II), N-butyl-7-fluoroquinolin-4-amine (III), $N^{4}$-(7-chlo-<smiles>CCN(CC)CCNc1ccnc2cc(F)ccc12</smiles>

I
II<smiles>CCCCNc1ccnc2cc(F)ccc12</smiles>

III<smiles>CCN(CC)CCCC(C)Nc1ccnc2cc(Cl)ccc12</smiles><smiles>[Y]C(CCCN)Nc1cc(OC)cc2cccnc12</smiles>

Fig. 1. Chemical structures of quinolines reported for anticancer activity:

I - $N^{1}$-(7-chloroquinolin-4-yl)- $N^{2}, N^{2}$-diethylethane-1,2-diamine

II $-N^{1}, N^{1}$-diethyl- $N^{2}$-(7-fluoroquinolin-4-yl)ethane-1,2-diamine

III - N-butyl-7-fluoroquinolin-4-amine

IV - $N^{4}$-(7-chloroquinolin-4-yl)- $N^{1}, N^{1}$-diethylpentane-1,4-diamine

V - $N^{4}$-(6-methoxyquinolin-8-yl)pentane-1,4-diamine 
roquinolin-4-yl)- $N^{1}, N^{1}$-diethylpentane-1,4-diamine (IV) and $N^{4}$-(6-methoxyquinolin-8-yl) pentane-1,4-diamine (primaquine, PQ, V) (Fig. 1) have been examined as cytotoxic agents against the MCF-7 breast cancer cell line. Some of these compounds were very effective (17). It has been reported that CQ and its analogs have a unique property of being accumulated in the lysosomes, raising intralysosomal $\mathrm{pH}$, and resulting in enhancement of cell killing by cancer therapeutic agents in a variety of different tumors (18). It is well known that many 2-pyridone derivatives revealed diverse biological activities such as anticancer (19), cardiotonic (20), and potential HIV-1 specific reverse transcriptase inhibiting (21). In view of the above mentioned findings and as a part of our research efforts to explore novel anticancer heterocyclic compounds (22-28), we have synthesized a new series of quinoline derivatives carrying a number of biologically active moieties as analogues to compounds I-V (Fig. 1).

\section{EXPERIMENTAL}

Melting points were uncorrected and determined in an open capillary on a Gallen Kamp melting point apparatus (Sanyo Gallen Kamp, UK). Precoated silica gel plates (Kieselgel 0.25 mm, 60 F254, Merck, Germany) were used for thin layer chromatography. A developing solvent system of chloroform/methanol (8:2) was used and the spots were detected by UV light. IR spectra (KBr disc) were recorded using an FT-IR spectrophotometer (Perkin Elmer, USA). ${ }^{1} \mathrm{H}$ NMR spectra were scanned on a NMR spectrophotometer (Bruker AXS Inc., Switzerland) operating at $500 \mathrm{MHz}$ for ${ }^{1} \mathrm{H}-$ and $125.76 \mathrm{MHz}$ for ${ }^{13} \mathrm{C}$. Chemical shifts are expressed in $\delta$-values (ppm) relative to TMS as an internal standard, using DMSO- $d_{6}$ as a solvent. Elemental analyses were done on a model 2400 CHNSO analyzer (Perkin Elmer, USA). All the values were within $\pm 0.4 \%$ of the theoretical values. All reagents used were of analytical grade. The starting material 3-aminoquinoline was purchased from Sigma (USA) and was directly used for the preparation of target compounds.

\section{Syntheses}

2-Cyano-N-(quinolin-3-yl)acetamide (1). - A mixture of quinolin-3-amine (1.44 g, 0.01 mol) and ethyl cyanoacetate $(1.13 \mathrm{~g}, 0.01 \mathrm{~mol})$ was fused at $220{ }^{\circ} \mathrm{C}$ for $2 \mathrm{~h}$. The reaction mixture was cooled and the obtained product was crystallized from ethanol to give 1.

4,6-Dimethyl-2-oxo-1-(quinolin-3-yl)-1,2-dihydropyridine-3-carbonitrile (4). - A mixture of compound $1(2.1 \mathrm{~g}, 0.01 \mathrm{~mol})$ and acetylacetone $(5 \mathrm{~mL})$ containing piperidine $(0.5 \mathrm{~mL})$ was fused for $6 \mathrm{~h}$. The reaction mixture was cooled and the obtained solid was recrystallized from dioxane to give 4 .

3-Amino-6-methyl-5-(quinolin-3-yl)thieno[3,4-c]pyridine-4(5H)-one (5). - A mixture of 4 $(2.75 \mathrm{~g}, 0.01 \mathrm{~mol})$ and elemental sulfur $(0.32 \mathrm{~g}, 0.01 \mathrm{~mol})$ in absolute ethanol (30 mL) containing 3 drops of triethylamine was refluxed for $8 \mathrm{~h}$. The obtained solid was crystallized from dioxane to give 5 .

8-Amino-3-methyl-1-oxo-6-phenyl-2-(quinolin-3-yl)-1,2-dihydroisoquinoline-7-carbonitrile (6), 8-amino-3-methyl-1-oxo-2-(quinolin-3-yl)-6-p-tolyl-1,2-dihydroisoquinoline-7-carbonitrile (7), 8-amino-6-(4-hydroxyphenyl)-3-methyl-1-oxo-2-(quinolin-3-yl)-1,2-dihydroisoquinoline-7-carbonitrile (8), 8-amino-6-(4-methoxyphenyl)-3-methyl-1-oxo-2-(quinolin-3-yl)-1,2-dihydroisoquinoline-7-car- 
M. M. Ghorab et al:: Novel quinolines carrying pyridine, thienopyridine, isoquinoline, thiazolidine, thiazole and thiophene moieties as potential anticancer agents, Acta Pharm. 66 (2016) 155-171.

bonitrile (9), 8-amino-3-methyl-1-oxo-2-(quinolin-3-yl)-6-stryl-1,2-dihydroisoquinoline-7-carbonitrile (10), 8-amino-6-(2-chlorophenyl)-3-methyl-1-oxo-2-(quinolin-3-yl)-1,2-dihydroisoquinoline-7-carbonitrile (11), 8-amino-6-(4-chlorophenyl)-3-methyl-1-oxo-2-(quinolin-3-yl)-1,2-dihydroisoquinoline7-carbonitrile (12), 8-amino-6-(4-(dimethylamino)phenyl)-3-methyl-1-oxo-2-(quinolin-3-yl)-1,2-dihydroisoquinoline-7-carbonitrile (13), 8-amino-6-(benzo[d][1,3]dioxol-5-yl)-3-methyl-1-oxo-2(quinolin-3-yl)-1,2-dihydroisoquinoline-7-carbonitrile (14), 8-amino-3-methyl-6-(3-nitrophenyl)-1oxo-2-(quinolin-3-yl)-1,2-dihydroisoquinoline-7-carbonitrile (15), 8-amino-3-methyl-6-(4nitrophenyl)-1-oxo-2-(quinolin-3-yl)-1,2-dihydroisoquinoline-7-carbonitrile (16), 8-amino-6-(2,4dichlorophenyl)-3-methyl-1-oxo-2-(quinolin-3-yl)-1,2-dihydroisoquinoline-7-carbonitrile (17), 8-amino-6-(2-methoxynaphthalen-1-yl)-3-methyl-1-oxo-2-(quinolin-3-yl)-1,2-dihydroisoquinoline7-carbonitrile (18), 8-amino-6-(4-methoxynaphthalen-1-yl)-3-methyl-1-oxo-2-(quinolin-3-yl)-1, 2-dihydroisoquinoline-7-carbonitrile (19), 8-amino-3-methyl-1-oxo-2-(quinolin-3-yl)-6-(thiophen-2yl)-1,2-dihydroisoquinoline-7-carbonitrile (20). General procedure. - A mixture of 4 (2.75 g, 0.01 $\mathrm{mol})$ and the respective benzylidenemalononitrile $(0.01 \mathrm{~mol})$ in ethanol $(20 \mathrm{~mL})$ containing a catalytic amount of piperidine $(0.5 \mathrm{~mL})$ was refluxed for $6 \mathrm{~h}$. The obtained solid was filtered and crystallized from dioxane to give 6-20, respectively (Scheme 1, Table I).

2-Cyano-3-(dimethylamino)-N-(quinolin-3-yl)acrylamide (21). - Dimethylformamide-dimethylacetal $(1.19 \mathrm{~g}, 0.01 \mathrm{~mol})$ was added to a solution of $\mathbf{1}(2.11 \mathrm{~g}, 0.01 \mathrm{~mol})$ in dry xylene $(20 \mathrm{~mL})$. The reaction mixture was refluxed for $8 \mathrm{~h}$, cooled and the obtained solid was recrystallized from ethanol to give 21.

2-(4-Oxothiazolidin-2-ylidene)-N-(quinolin-3-yl) acetamide (22). - A mixture of $\mathbf{1}$ (2.11 g, $0.01 \mathrm{~mol})$ and 2-sulfanylacetic acid $(0.92 \mathrm{~g}, 0.01 \mathrm{~mol})$ in dry pyridine $(10 \mathrm{~mL})$ was refluxed for $18 \mathrm{~h}$. The reaction mixture was cooled and poured into ice/water. The obtained solid was recrystallized from acetic acid to give 22 .

4-Amino-3-phenyl-N-(quinolin-3-yl)-2-thioxo-2,3-dihydrothiazole-5-carboxamide (23), 4-amino-N-(quinolin-3-yl)-2-thioxo-3-p-tolyl-2,3-dihydrothiazole-5-carboxamide (24), 4-amino3-(4-fluorophenyl)-N-(quinolin-3-yl)-2-thioxo-2,3-dihydrothiazole-5-carboxamide (25), 4-amino3-(4-methoxyphenyl)-N-(quinolin-3-yl)-2-thioxo-2,3-dihydrothiazole-5-carboxamide (26), 4-amino-3-(4-nitrophenyl)-N-(quinolin-3-yl)-2-thioxo-2,3-dihydrothiazole-5-carboxamide (27), 4-amino-3-(4-bromophenyl)-N-(quinolin-3-yl)-2-thioxo-2,3-dihydrothiazole-5-carboxamide (28), 4-amino-3-(4-iodophenyl)-N-(quinolin-3-yl)-2-thioxo-2,3-dihydrothiazole-5-carboxamide (29). General procedure. - To a suspension of 1 (2.11 g, $0.01 \mathrm{~mol})$ in absolute ethanol $(20 \mathrm{~mL})$, finely divided sulfur $(0.32 \mathrm{~g}, 0.01 \mathrm{~mol}), 3$ drops of triethylamine, aryl isothiocyanate $(0.01$ $\mathrm{mol})$ and DMF $(10 \mathrm{~mL})$ were added. The reaction mixture was stirred at $60{ }^{\circ} \mathrm{C}$ for $6 \mathrm{~h}$, then left to cool at room temperature. The separated product was filtered, washed with ethanol, dried and crystallized from DMF/ethanol (2:1) to give 23-29, respectively.

2-Amino-N-(quinolin-3-yl)-5,6-dihydro-4H-cyclopenta[b]thiophene-3-carboxamide (33), 2-amino-N-(quinolin-3-yl)-4,5,6,7-tetrahydrobenzo[b]thiophene-3-carboxamide (34), 2-amino-N(quinolin-3-yl)-5,6,7,8-tetrahydro-4H-cyclohepta[b] thiophene-3-carboxamide (35). General procedure. - To a solution of $\mathbf{1}(2.11 \mathrm{~g}, 0.01 \mathrm{~mol})$ in absolute ethanol $(30 \mathrm{~mL})$, morpholine $(1 \mathrm{~mL})$, cyclopentanone or cyclohexanone and/or cycloheptanone $(0.01 \mathrm{~mol})$ and sulfur $(0.32 \mathrm{~g}, 0.01$ mol) were added. The reaction mixture was refluxed for $8 \mathrm{~h}$. The separated product was filtered, dried and crystallized from dioxane to give 33-35, respectively. 
M. M. Ghorab et al:: Novel quinolines carrying pyridine, thienopyridine, isoquinoline, thiazolidine, thiazole and thiophene moieties as potential anticancer agents, Acta Pharm. 66 (2016) 155-171.

Table I. Physical and analytical data of the newly synthesized compounds

\begin{tabular}{|c|c|c|c|c|c|c|}
\hline \multirow{2}{*}{$\begin{array}{c}\text { Compd. } \\
\text { No. }\end{array}$} & \multirow{2}{*}{$\begin{array}{c}\text { Formula } \\
\left(M_{\mathrm{r}}\right)\end{array}$} & \multirow{2}{*}{$\begin{array}{l}\text { M. p. } \\
\left({ }^{\circ} \mathrm{C}\right)\end{array}$} & \multirow{2}{*}{$\begin{array}{c}\text { Yield } \\
(\%)\end{array}$} & \multicolumn{3}{|c|}{ Analysis (calcd./found) (\%) } \\
\hline & & & & $\mathrm{C}$ & $\mathrm{H}$ & $\mathrm{N}$ \\
\hline 1 & $\mathrm{C}_{12} \mathrm{H}_{9} \mathrm{~N}_{3} \mathrm{O}(211.22)$ & 220.4 & 92 & $68.24 / 68.51$ & $4.29 / 4.08$ & $19.89 / 19.59$ \\
\hline 4 & $\mathrm{C}_{17} \mathrm{H}_{13} \mathrm{~N}_{3} \mathrm{O}(275.30)$ & 278.7 & 86 & $74.17 / 74.46$ & $4.76 / 4.44$ & $15.26 / 15.51$ \\
\hline 5 & $\mathrm{C}_{17} \mathrm{H}_{13} \mathrm{~N}_{3} \mathrm{OS}$ (307.37) & 199.9 & 68 & $66.43 / 66.64$ & $4.26 / 4.51$ & $13.67 / 13.39$ \\
\hline 6 & $\mathrm{C}_{26} \mathrm{H}_{18} \mathrm{~N}_{4} \mathrm{O}(402.45)$ & 147.2 & 81 & $77.59 / 77.27$ & $4.51 / 4.19$ & $13.92 / 13.65$ \\
\hline 7 & $\mathrm{C}_{27} \mathrm{H}_{20} \mathrm{~N}_{4} \mathrm{O}(416.47)$ & 97.7 & 77 & $77.87 / 77.56$ & $4.84 / 4.59$ & $13.45 / 13.24$ \\
\hline 8 & $\mathrm{C}_{26} \mathrm{H}_{18} \mathrm{~N}_{4} \mathrm{O}_{2}(418.45)$ & 269.9 & 83 & $74.63 / 74.31$ & $4.34 / 4.12$ & $13.39 / 13.11$ \\
\hline 9 & $\mathrm{C}_{27} \mathrm{H}_{20} \mathrm{~N}_{4} \mathrm{O}_{2}(432.47)$ & 119.8 & 79 & $74.98 / 74.63$ & $4.66 / 4.29$ & $12.95 / 13.18$ \\
\hline 10 & $\mathrm{C}_{28} \mathrm{H}_{20} \mathrm{~N}_{4} \mathrm{O}(428.48)$ & 245.2 & 68 & $78.49 / 78.27$ & $4.70 / 4.43$ & $13.08 / 13.36$ \\
\hline 11 & $\mathrm{C}_{26} \mathrm{H}_{17} \mathrm{~N}_{4} \mathrm{O}(436.89)$ & 156.7 & 78 & $71.48 / 71.26$ & $3.92 / 3.65$ & $12.82 / 12.49$ \\
\hline 12 & $\mathrm{C}_{26} \mathrm{H}_{17} \mathrm{ClN}_{4} \mathrm{O}(436.89)$ & 288.8 & 68 & $71.48 / 71.16$ & $3.92 / 3.63$ & $12.82 / 13.11$ \\
\hline 13 & $\mathrm{C}_{28} \mathrm{H}_{23} \mathrm{~N}_{5} \mathrm{O}(445.52)$ & 281.8 & 63 & $75.49 / 75.20$ & $5.20 / 5.01$ & $15.72 / 15.38$ \\
\hline 14 & $\mathrm{C}_{27} \mathrm{H}_{18} \mathrm{~N}_{4} \mathrm{O}_{3}(446.46)$ & 318.0 & 74 & $72.64 / 72.33$ & $4.06 / 4.27$ & $12.55 / 12.29$ \\
\hline 15 & $\mathrm{C}_{26} \mathrm{H}_{17} \mathrm{~N}_{5} \mathrm{O}_{3}$ (447.44) & 150.8 & 67 & $69.79 / 69.52$ & $3.83 / 3.48$ & $15.65 / 15.41$ \\
\hline 16 & $\mathrm{C}_{26} \mathrm{H}_{17} \mathrm{~N}_{5} \mathrm{O}_{3}(447.44)$ & 234.0 & 77 & $69.79 / 69.43$ & $3.83 / 3.50$ & $15.65 / 15.34$ \\
\hline 17 & $\mathrm{C}_{26} \mathrm{H}_{16} \mathrm{Cl}_{2} \mathrm{~N}_{4} \mathrm{O}(471.34)$ & 174.2 & 78 & $69.25 / 69.59$ & $3.42 / 3.12$ & $11.89 / 12.24$ \\
\hline 18 & $\mathrm{C}_{31} \mathrm{H}_{22} \mathrm{~N}_{4} \mathrm{O}_{2}(482.53)$ & 215.9 & 59 & $77.16 / 77.52$ & $4.60 / 4.33$ & $11.61 / 11.30$ \\
\hline 19 & $\mathrm{C}_{31} \mathrm{H}_{22} \mathrm{~N}_{4} \mathrm{O}_{2}(482.53)$ & 344.9 & 63 & 77.16/77.46 & $4.60 / 4.91$ & $11.61 / 11.35$ \\
\hline 20 & $\mathrm{C}_{24} \mathrm{H}_{16} \mathrm{~N}_{5} \mathrm{OS}(408.48)$ & 128.9 & 74 & $70.57 / 70.88$ & $3.95 / 3.76$ & $13.72 / 13.42$ \\
\hline 21 & $\mathrm{C}_{15} \mathrm{H}_{14} \mathrm{~N}_{4} \mathrm{O}(266.30)$ & 187.9 & 85 & $67.65 / 67.36$ & $5.30 / 5.10$ & $21.04 / 21.31$ \\
\hline 22 & $\mathrm{C}_{16} \mathrm{H}_{11} \mathrm{~N}_{3} \mathrm{O}_{2} \mathrm{~S}(285.32)$ & 296.6 & 78 & $58.93 / 58.66$ & $3.89 / 3.60$ & $14.73 / 14.39$ \\
\hline 23 & $\mathrm{C}_{19} \mathrm{H}_{14} \mathrm{~N}_{4} \mathrm{OS}_{2}(378.47)$ & 97.0 & 88 & $60.30 / 60.06$ & $3.73 / 3.48$ & $14.80 / 14.52$ \\
\hline 24 & $\mathrm{C}_{20} \mathrm{H}_{16} \mathrm{~N}_{4} \mathrm{OS}_{2}(392.50)$ & 112.3 & 85 & $61.20 / 61.49$ & $4.11 / 4.41$ & $14.27 / 14.53$ \\
\hline 25 & $\mathrm{C}_{19} \mathrm{H}_{13} \mathrm{FN}_{4} \mathrm{OS}_{2}(396.46)$ & 263.1 & 71 & $57.56 / 57.32$ & $3.31 / 3.07$ & $14.13 / 14.40$ \\
\hline 26 & $\mathrm{C}_{20} \mathrm{H}_{16} \mathrm{~N}_{4} \mathrm{O}_{2} \mathrm{~S}_{2}(408.50)$ & 275.4 & 78 & $58.80 / 58.54$ & $3.95 / 3.63$ & $13.72 / 13.45$ \\
\hline 27 & $\mathrm{C}_{19} \mathrm{H}_{13} \mathrm{~N}_{3} \mathrm{O}_{3} \mathrm{~S}_{2}(423.47)$ & 169.9 & 70 & $53.89 / 53.55$ & $3.09 / 3.37$ & $16.45 / 16.16$ \\
\hline 28 & $\mathrm{C}_{19} \mathrm{H}_{13} \mathrm{BrN}_{4} \mathrm{OS}_{2}(457.37)$ & 148.6 & 80 & $49.90 / 49.61$ & $2.86 / 2.48$ & $12.25 / 12.01$ \\
\hline 29 & $\mathrm{C}_{19} \mathrm{H}_{13} \mathrm{IN}_{4} \mathrm{OS}_{2}(504.37)$ & 138.7 & 79 & $45.25 / 45.51$ & $2.60 / 2.32$ & $11.11 / 11.40$ \\
\hline 33 & $\mathrm{C}_{17} \mathrm{H}_{15} \mathrm{~N}_{3} \mathrm{OS}$ (309.39) & 230.0 & 66 & $66.00 / 66.29$ & $4.89 / 4.57$ & $13.58 / 13.88$ \\
\hline 34 & $\mathrm{C}_{18} \mathrm{H}_{17} \mathrm{~N}_{3} \mathrm{OS}$ (323.41) & 197.3 & 69 & $66.85 / 66.49$ & $5.30 / 5.04$ & $12.99 / 12.66$ \\
\hline 35 & $\mathrm{C}_{19} \mathrm{H}_{19} \mathrm{~N}_{3} \mathrm{OS}(337.44)$ & 161.1 & 61 & $67.63 / 67.37$ & $5.68 / 5.35$ & $12.45 / 12.19$ \\
\hline
\end{tabular}

Physicochemical and spectral data are given in Tables I and II and synthetic pathways in Schemes 1 and 2. 
Table II. Spectral data for compounds

\begin{tabular}{lll}
\hline Dं IR & ${ }^{1} \mathrm{H}$ NMR (DMSO- $\left.d_{6}\right)$ & Mass \\
हैं $\left(v_{\max } \mathrm{cm}^{-1}\right)$ & ${ }^{13} \mathrm{CNMR}\left(\mathrm{DMSO}-d_{6}\right)$ & $(\mathrm{m} / \mathrm{z}, \%)$ \\
0 & $(\delta, \mathrm{ppm})$ & \\
\hline
\end{tabular}

$13260(\mathrm{NH}), 3091$ ( $\mathrm{CH}$ arom.), $4.0\left(\mathrm{~s}, 2 \mathrm{H}, \mathrm{CH}_{2}\right), 7.5,8.6$ (2s, 2H, 2CH quinoline), 7.6-7.9 2957, 2927 (CH aliph.), $\quad(\mathrm{m}, 4 \mathrm{H}, \mathrm{Ar}-\mathrm{H}), 10.8$ (s, 1H, NH, $\mathrm{D}_{2} \mathrm{O}-$ exchangeable) $2201(\mathrm{C} \equiv \mathrm{N}), 1700(\mathrm{C}=\mathrm{O}), \quad 24.7,115.6,122.6,123.8,125.4,126.3,127.7,128.5,132.0$, $211\left[\mathrm{M}^{+}\right]$ $1617(\mathrm{C}=\mathrm{N})$ $144.1,144.4,161.9$

43062 (CH arom.), 2937, $28702.0\left(\mathrm{~s}, 6 \mathrm{H}, 2 \mathrm{CH}_{3}\right), 6.5$ (s, $1 \mathrm{H}, \mathrm{CH}$ pyridone), 7.3, 8.8 ( $\mathrm{CH}$ aliph.), $2218(\mathrm{C} \equiv \mathrm{N})$, ( $2 \mathrm{~s}, 2 \mathrm{H}, 2 \mathrm{CH}$ quinoline), $7.5-8.1(\mathrm{~m}, 4 \mathrm{H}, \mathrm{Ar}-\mathrm{H})$ (9.4), 68 $1658(\mathrm{C}=\mathrm{O}), 1585(\mathrm{C}=\mathrm{N})$ 20.7, 21.7, 100.0, 109.3, 115.6, 127.3, 127.5, 128.4, 128.8, (100) $130.7,130.8,135.1,147.0,149.5,152.1,160.2,160.8$

$53406,3326\left(\mathrm{NH}_{2}\right), 2976,28361.6\left(\mathrm{~s}, 1 \mathrm{H}, \mathrm{CH}_{3}\right), 5.1(\mathrm{~s}, 1 \mathrm{H}, \mathrm{CH}$ pyridone), $6.6(\mathrm{~s}, 1 \mathrm{H}, \mathrm{CH}$ (CH aliph.), 1657 ( $\mathrm{C}=\mathrm{O})$, $1611(\mathrm{C}=\mathrm{N})$ thiophene), 7.1, $8.6(2 \mathrm{~s}, 2 \mathrm{H}, 2 \mathrm{CH}$ quinoline), $7.2-8.3(\mathrm{~m}$, $6 \mathrm{H}, \mathrm{Ar}-\mathrm{H}+\mathrm{NH}_{2}$ ) 21.7, 105.4, 123.6, 124.1, 126.2 (2), 127.1 (2), 128.6, 131.8, 134.7, 136.0, 138.2, 141.0, 142.5, 151.2, 161.2

$63405,3386\left(\mathrm{NH}_{2}\right), 3066$ (CH arom.), 2910, 2861 ( $\mathrm{CH}$ aliph.), $2184(\mathrm{C} \equiv \mathrm{N})$, $1695(\mathrm{C}=\mathrm{O}), 1586(\mathrm{C}=\mathrm{N})$

$73310,3205\left(\mathrm{NH}_{2}\right), 3100$ (CH arom.), 2920, 2863 (CH aliph.), $2184(\mathrm{C} \equiv \mathrm{N})$, $1689(\mathrm{C}=\mathrm{O}), 1599(\mathrm{C}=\mathrm{N})$

83431 (br, OH), 3371, 3312 $\left(\mathrm{NH}_{2}\right), 3069$ (CH arom.), 2927, 2863 ( $\mathrm{CH}$ aliph.), $2217(\mathrm{C} \equiv \mathrm{N}), 1661(\mathrm{C}=\mathrm{O})$, $1585(\mathrm{C}=\mathrm{N})$

$93378,3296\left(\mathrm{NH}_{2}\right), 3061$ (CH arom.), 2971, 2819 (CH aliph.), $2191(\mathrm{C} \equiv \mathrm{N})$, $1676(\mathrm{C}=\mathrm{O}), 1599(\mathrm{C}=\mathrm{N})$

$103391,3286\left(\mathrm{NH}_{2}\right), 3100$ (CH arom.), 2976, 2912 (CH aliph.), $2218(\mathrm{C} \equiv \mathrm{N})$, $1663(\mathrm{C}=\mathrm{O}), 1609(\mathrm{C}=\mathrm{N})$ $1.7\left(\mathrm{~s}, 3 \mathrm{H}, \mathrm{CH}_{3}\right), 5.2\left(\mathrm{~s}, 2 \mathrm{H}, \mathrm{NH}_{2}, \mathrm{D}_{2} \mathrm{O}\right.$-exchangeable), 6.4 (s, $1 \mathrm{H}, \mathrm{CH}$ pyridone), 7.1, $8.6(2 \mathrm{~s}, 2 \mathrm{H}, 2 \mathrm{CH}$ quinoline), 7.2-8.3 (m, 10H, Ar-H)

21.6, 95.2, 101.8, 114.2, 116.7, 116.9, 122.8, 123.6, 124.7, $127.5,127.9,128.6(2), 128.9,129.1,129.2$ (2), 129.4, 135.1 (2), $138.4,139.3,142.6,144.8,148.1,157.4$

$1.3\left(\mathrm{~s}, 3 \mathrm{H}, \mathrm{CH}_{3}\right), 2.3\left(\mathrm{~s}, 3 \mathrm{H}, \mathrm{CH}_{3}\right.$ tolyl), $6.0\left(\mathrm{~s}, 2 \mathrm{H}, \mathrm{NH}_{2}, \quad 416\left[\mathrm{M}^{+}\right]\right.$ $\mathrm{D}_{2} \mathrm{O}$-exchangeable), 6.5 (s, $1 \mathrm{H}, \mathrm{CH}$ pyridone), 7.0, 8.6 (2s, (24.7), 77 $2 \mathrm{H}, 2 \mathrm{CH}$ quinoline), $7.2-8.4(\mathrm{~m}, 9 \mathrm{H}, \mathrm{Ar}-\mathrm{H})$

18.4, 24.0, 97.4, 100.4, 116.6, 116.8, 117.9, 125.2 (2), 127.4, 127.9, 129.1 (2), 128.5, 128.8, 129.3 (2), 129.7, 132.3, 135.8, 137.3, 138.3, 139.7, $145.2(2), 149.7,158.3$

$2.0\left(\mathrm{~s}, 3 \mathrm{H}, \mathrm{CH}_{3}\right), 6.0\left(\mathrm{~s}, 2 \mathrm{H}, \mathrm{NH}_{2}, \mathrm{D}_{2} \mathrm{O}\right.$-exchangeable), 6.5 (s, $1 \mathrm{H}, \mathrm{CH}$ pyridone), $7.0,8.8(2 \mathrm{~s}, 2 \mathrm{H}, 2 \mathrm{CH}$ quinoline), 7.7-8.5 (m, 9H, Ar-H), 9.0 (s, 1H, OH, $\mathrm{D}_{2} \mathrm{O}$-exchangeable) $163(100)$ 20.7, 92.4, 100.0, 115.6, 116.8 (2), 118.1, 118.3, 123.7, 126.1, $127.3,127.5$ (2), 128.4 (2), 128.8 (2), 130.7, 135.1 (2), 139.6, $147.0(2), 149.5,152.1,1602$

$1.6\left(\mathrm{~s}, 3 \mathrm{H}, \mathrm{CH}_{3}\right), 3.8\left(\mathrm{~s}, 3 \mathrm{H}, \mathrm{OCH}_{3}\right), 6.2\left(\mathrm{~s}, 2 \mathrm{H}, \mathrm{NH}_{2}, \quad 432\left[\mathrm{M}^{+}\right]\right.$ $\mathrm{D}_{2} \mathrm{O}$-exchangeable), 6.3 (s, $1 \mathrm{H}, \mathrm{CH}$ pyridone), $6.9,6.8$ (2s, (45.9), 56 $2 \mathrm{H}, 2 \mathrm{CH}$ quinoline), 7.1-8.4 (m, 9H, Ar-H)

18.4, 56.0, 96.5, 99.9, 113.7 (2), 114.4, 115.1, 116.0, 125.2,

$127.3,127.5,127.7,128.1,128.4,128.8$ (2), 129.6, 130.0, 135.6, $138.0,140.2,146.9(2), 149.9,153.9,160.5$

$1.5\left(\mathrm{~s}, 3 \mathrm{H}, \mathrm{CH}_{3}\right), 6.2\left(\mathrm{~s}, 2 \mathrm{H}, \mathrm{NH}_{2}, \mathrm{D}_{2} \mathrm{O}\right.$-exchangeable), $6.4 \quad 428\left[\mathrm{M}^{+}\right]$ (s, $1 \mathrm{H}, \mathrm{CH}$ pyridone), 6.8, $7.3(2 \mathrm{~d}, 2 \mathrm{H}, \mathrm{CH}=\mathrm{CH}, J=7.4 \mathrm{~Hz}), \quad(44.7), 93$ $7.4,8.8(2 \mathrm{~s}, 2 \mathrm{H}, 2 \mathrm{CH}$ quinoline), $7.5-8.5(\mathrm{~m}, 10 \mathrm{H}, \mathrm{Ar}-\mathrm{H})$ 20.7, 97.6, 100.0, 109.3, $115.6(2), 123.6,123.8,124.1,124.4$, 125.0, 126.8, 127.1 (2), 127.6 (2), 128.3 (2), 128.9 (2), 135.1 (2), $143.2(2), 147.0(2), 149.5,152.1$ 
$113398,3334\left(\mathrm{NH}_{2}\right), 3077$ (CH arom.), 2929, 2845 (CH aliph.), $2219(\mathrm{C} \equiv \mathrm{N})$ $1653(\mathrm{C}=\mathrm{O}), 1576(\mathrm{C}=\mathrm{N})$, 754 (C-Cl)

$123265,3212\left(\mathrm{NH}_{2}\right), 3091$ (CH arom.), 2976, 2844 (CH aliph.), $2218(\mathrm{C} \equiv \mathrm{N})$, $1658(\mathrm{C}=\mathrm{O}), 1587(\mathrm{C}=\mathrm{N})$, 818 (C-Cl)

13 3406, $3399\left(\mathrm{NH}_{2}\right), 3047$ (CH arom.), $2221(\mathrm{C} \equiv \mathrm{N})$, $1654(\mathrm{C}=\mathrm{O}), 1592(\mathrm{C}=\mathrm{N})$

$143368,3276\left(\mathrm{NH}_{2}\right), 3050$ (CH arom.), 2921, 2898 (CH aliph.), $2215(\mathrm{C} \equiv \mathrm{N})$, $1659(\mathrm{C}=\mathrm{O}), 1597(\mathrm{C}=\mathrm{N})$

15 3431, $3291\left(\mathrm{NH}_{2}\right), 3100$ (CH arom.), 2946, 2908 (CH aliph.), $2200(\mathrm{C} \equiv \mathrm{N})$, $1653(\mathrm{C}=\mathrm{O}), 1595(\mathrm{C}=\mathrm{N})$, $1568,1361\left(\mathrm{NO}_{2}\right)$

16 3386, $3351\left(\mathrm{NH}_{2}\right), 3081$ (CH arom.), 2917, 2866 (CH aliph.), $2189(\mathrm{C} \equiv \mathrm{N})$, $1654(\mathrm{C}=\mathrm{O}), 1585(\mathrm{C}=\mathrm{N})$, $1518,1343\left(\mathrm{NO}_{2}\right)$

17 3391, $3317\left(\mathrm{NH}_{2}\right), 3100$ (CH arom.), 2929, 2810 (CH aliph.), $2216(\mathrm{C} \equiv \mathrm{N})$, $1660(\mathrm{C}=\mathrm{O}), 1585(\mathrm{C}=\mathrm{N})$, $751(\mathrm{C}-\mathrm{Cl})$

18 3436, $3421\left(\mathrm{NH}_{2}\right), 3058$ (CH arom.), 2934, 2836 (CH aliph.), $2213(\mathrm{C} \equiv \mathrm{N})$, $1654(\mathrm{C}=\mathrm{O}), 1578(\mathrm{C}=\mathrm{N})$ $1.5\left(\mathrm{~s}, 3 \mathrm{H}, \mathrm{CH}_{3}\right), 5.9\left(\mathrm{~s}, 2 \mathrm{H}, \mathrm{NH}_{2}, \mathrm{D}_{2} \mathrm{O}\right.$ - exchangeable), $6.5437\left[\mathrm{M}^{+}\right]$ (s, $1 \mathrm{H}, \mathrm{CH}$ pyridone), 7.2, 8.8 (2s, 2H, 2CH quinoline), (19.8), 76 7.4-8.1 (m, 9H, Ar-H) 20.7, 88.1, 100.1, 115.6 (2), 115.9, 126.9 (2), 127.3, 127.5, 127.7, $128.4,128.8,129.3,129.7$ (2), 130.0, 131.3, 133.2, 135.1 (2), $142.3,147.0,149.5,152.1,160.2$

$1.6\left(\mathrm{~s}, 3 \mathrm{H}, \mathrm{CH}_{3}\right), 4.4\left(\mathrm{~s}, 2 \mathrm{H}, \mathrm{NH}_{2}, \mathrm{D}_{2} \mathrm{O}\right.$-exchangeable), 6.5 (s, $1 \mathrm{H}, \mathrm{CH}$ pyridone), 7.0, 8.8 (2s, $2 \mathrm{H}, 2 \mathrm{CH}$ quinoline), 7.2-8.3 (m, 9H, Ar-H) 22.1, 85.2, 101.6, 115.8, 116.0 (2), 122.7, 123.4, 124.8, 126.1, $128.6,128.7,129.8$ (2), 130.3 (2), 131.6, 131.9, 134.3 (2), 136.1, $142.7,143.6,149.1(2), 157.8$

$2.0\left(\mathrm{~s}, 3 \mathrm{H}, \mathrm{CH}_{3}\right), 2.9\left(\mathrm{~s}, 6 \mathrm{H}, \mathrm{N}\left(\mathrm{CH}_{3}\right)_{2}\right), 6.0\left(\mathrm{~s}, 2 \mathrm{H}, \mathrm{NH}_{2}, \quad 446\left[\mathrm{M}^{+}\right]\right.$ $\mathrm{D}_{2} \mathrm{O}$-exchangeable), 6.1 (s, 1H, CH pyridone), 7.0, 8.6 (2s, (13.9), 91 $2 \mathrm{H}, 2 \mathrm{CH}$ quinoline), 7.2-8.2 (m, 9H, Ar-H)

(100) 21.9, 40.1 (2), 95.5, 102.6, 111.7 (2), 112.0, 115.4, 116.0, 122.2, 127.3 (2), 127.4 (2), 128.4, 128.8 (2), 129.8, 130.7, 135.2, 141.3 (2), 146.9 (2), 149.8, 150.5, 154.8

$2.0\left(\mathrm{~s}, 3 \mathrm{H}, \mathrm{CH}_{3}\right), 6.0$ (s, 2H, $\mathrm{NH}_{2}, \mathrm{D}_{2} \mathrm{O}$-exchangeable), 6.1 (s, 1H, CH pyridone), 6.5 (s, 2H, O-CH $\left.\mathrm{CH}_{2}-\mathrm{O}\right), 7.0,8.6$ (2s, 2H, 2CH quinoline), 7.2-8.2 (m, 8H, Ar-H)

$436\left[\mathrm{M}^{+}\right]$

(1.8), 227

(100) 20.7, 94.3, 100.8, 101.6, 109.3, 116.8 (2), 118.3, 118.7, 121.9, 122.4, 124.6, 127.3, 127.5, 128.4, 128.8, 130.7 (2), 135.1 (2), 142.0, 147.0, 149.0 (2), 152.1 (2), 157.2

$1.6\left(\mathrm{~s}, 3 \mathrm{H}, \mathrm{CH}_{3}\right), 5.8\left(\mathrm{~s}, 2 \mathrm{H}, \mathrm{NH}_{2}, \mathrm{D}_{2} \mathrm{O}\right.$-exchangeable), 6.5 (s, $1 \mathrm{H}, \mathrm{CH}$ pyridone), 7.5, 8.8 (2s, $2 \mathrm{H}, 2 \mathrm{CH}$ quinoline), 7.6-8.2 (m, 8H, Ar-H) 20.7, 95.7, 100.0, 109.3, 115.6 (2), 122.1, 123.3 (2), 127.3 (2), $127.5,128.4,128.7,130.4,130.7,130.8,135.1(2), 140.8$ (2), $142.6,147.0,149.5$ (2), 160.2

$1.6\left(\mathrm{~s}, 3 \mathrm{H}, \mathrm{CH}_{3}\right), 6.0$ (s, 2H, $\mathrm{NH}_{2}, \mathrm{D}_{2} \mathrm{O}$-exchangeable), 6.5 (s, $1 \mathrm{H}, \mathrm{CH}$ pyridone), 7.2, 8.8 (2s, 2H, 2CH quinoline), 7.4-8.5 (m, 9H, Ar-H)

$18.5,94.6,100.0,109.3,115.6$ (2), 123.6 (2), 124.2 (2), 127.3, $127.5,128.4,128.7$ (2), 130.1, 130.4, 135.1 (2), 142.6, 144.8 (2), 147.0 (2), 149.5, 152.1

1.5 (s, 3H, $\left.\mathrm{CH}_{3}\right), 6.0$ (s, 2H, $\mathrm{NH}_{2}, \mathrm{D}_{2} \mathrm{O}$-exchangeable), 6.9 (s, 1H, CH pyridone), 7.3, 8.8 (2s, 2H, 2CH quinoline), 7.4-8.5 (m, 8H, Ar-H) 18.5, 93.6, 100.0, 109.3, 115.6 (2), 127.3 (2), 127.5 (2), 128.1, 128.4, 128.7, 129.4, 130.7, 130.8, 132.2 (2), 135.7 (2), 142.1 (2), 147.0 (2), 149.5, 157.4

$2.0\left(\mathrm{~s}, 3 \mathrm{H}, \mathrm{CH}_{3}\right), 3.8\left(\mathrm{~s}, 3 \mathrm{H}, \mathrm{OCH}_{3}\right), 6.0\left(\mathrm{~s}, 2 \mathrm{H}, \mathrm{NH}_{2}\right.$, $\mathrm{D}_{2} \mathrm{O}$-exchangeable), 6.5 (s, $1 \mathrm{H}, \mathrm{CH}$ pyridone), $7.2,8.8$ (2s, 2H, 2CH quinoline), 7.4-8.4 (m, 11H, Ar-H) $446\left[\mathrm{M}^{+}\right]$ (6.9), 109 (100)

$447\left[\mathrm{M}^{+}\right]$ (15.6), 68 (100)

$447\left[\mathrm{M}^{+}\right]$ (28.3), 74 (100)

$471\left[\mathrm{M}^{+}\right]$ (17.1), 58 (100)

$18.5,56.0,98.1,100.0,113.7,115.6,116.1,122.9,123.9$ (2), $127.1,127.3,127.5,127.6,128.4$ (2), 128.7 (2), 130.5 (2), 130.7 (2), 133.0, 135.6 (2), 142.3, 147.0 (2), 149.5, 156.3, 160.2
$483\left[\mathrm{M}^{+}\right]$

(68.6), 286 (100) 
$193404,3395\left(\mathrm{NH}_{2}\right), 3100$ (CH arom.), 2961, 2846 (CH aliph.), $2214(\mathrm{C} \equiv \mathrm{N})$, $1653(\mathrm{C}=\mathrm{O}), 1589(\mathrm{C}=\mathrm{N})$

$203401,3381\left(\mathrm{NH}_{2}\right), 3066$ (CH arom.), 2972, 2818 (CH aliph.), $2190(\mathrm{C} \equiv \mathrm{N})$, $1688(\mathrm{C}=\mathrm{O}), 1508(\mathrm{C}=\mathrm{N})$ $1.8\left(\mathrm{~s}, 3 \mathrm{H}, \mathrm{CH}_{3}\right), 4.0\left(\mathrm{~s}, 3 \mathrm{H}, \mathrm{OCH}_{3}\right), 6.0\left(\mathrm{~s}, 2 \mathrm{H}, \mathrm{NH}_{2}\right.$ $\mathrm{D}_{2} \mathrm{O}$-exchangeable), 6.5 (s, 1H, CH pyridone), 7.0, 8.5 (2s, 2H, 2CH quinoline), 7.2-8.3 (m, 11H, Ar-H) 20.7, 57.4, 92.1, 100.0, 109.3, 113.2, 115.6, 117.8, 119.9, 122.8, 123.6, 124.4, 124.8, 124.9, 125.0, 126.3, 127.3, 127.5, 128.4, $128.8,130.7$ (2), 130.8, 135.1, 138.5, 139.6 (2), 147.0, 149.5, $152.1(2)$

$1.8\left(\mathrm{~s}, 3 \mathrm{H}, \mathrm{CH}_{3}\right), 5.9$ (s, 2H, $\mathrm{NH}_{2}, \mathrm{D}_{2} \mathrm{O}$-exchangeable), 6.7 (s, $1 \mathrm{H}, \mathrm{CH}$ pyridone), 7.0, 8.6 (2s, $2 \mathrm{H}, 2 \mathrm{CH}$ quinoline), 7.2-8.2 (m, 8H, Ar-H)

18.5, 97.1, 102.8, 109.3, 115.6 (2), 120.1, 127.3 (2), 127.5 (2), $128.4,128.8,129.9$ (2), 130.7, 130.8, 133.5, 135.1, 140.2 (2), $142.6,149.6,153.7$

213287 (NH), 3079 (CH arom.), 2.6 (s, 6H, 2 $\left.\mathrm{CH}_{3}\right), 7.0-8.3$ (m, 4H, Ar-H), 7.5 (s.1H, CH), 2936, 2871 (CH aliph.), $2224(\mathrm{C} \equiv \mathrm{N}), 1688(\mathrm{C}=\mathrm{O})$, $1593(\mathrm{C}=\mathrm{N})$
7.3, 8.6 (2s, 2H, 2CH quinoline), $8.9(\mathrm{~s}, 1 \mathrm{H}, \mathrm{NH}$,

$\mathrm{D}_{2} \mathrm{O}$-exchangeable)

40.0 (2), 90.0, 118.8, $125.6(2), 127.2,127.5,128.2,130.2$, 135.5 (2), 146.5, 154.9, 162.3

223159 (NH), 3100 (CH arom.), 3.7 (s, 2H, CH $\mathrm{CH}_{2} \mathrm{CO}$, 5.8 (s, 1H, CH), 7.5, 8.8 (2s, 2H, 2CH 2966, 2871 (CH aliph.), $1705,1679(2 \mathrm{C}=\mathrm{O})$, $1581(\mathrm{C}=\mathrm{N})$ quinoline), 7.6-8.0 (m, 4H, Ar-H), 10.3 (s, 1H, NH, $\mathrm{D}_{2} \mathrm{O}$-exchangeable), $11.6(\mathrm{~s}, 1 \mathrm{H}, \mathrm{NH}$, thiazolidine, $\mathrm{D}_{2} \mathrm{O}$-exchangeable) $32.1,91.9,120.7,126.9,127.3,127.5,128.0,128.4,133.5,143.7$, $144.1,155.6,165.9,174.1$

23 3410, $3286\left(\mathrm{NH}, \mathrm{NH}_{2}\right)$, 3066 (CH arom.), 1686 $(\mathrm{C}=\mathrm{O}), 1577(\mathrm{C}=\mathrm{N})$, $1234(\mathrm{C}=\mathrm{S})$

4.5 (s, 2H, $\mathrm{NH}_{2}, \mathrm{D}_{2} \mathrm{O}$-exchangeable), 6.9, 8.7 (2s, 2H, 2CH $378\left[\mathrm{M}^{+}\right]$ quinoline), 7.1-8.2 (m, 9H, Ar-H), 11.0 (s, 1H, NH, $\mathrm{D}_{2} \mathrm{O}$-exchangeable)

(12.8), 91 81.9, 123.1 (2), 124.3, 124.5, 127.4 (2), 127.7, 128.1, 129.0, 129.1 (2), 133.4, 135.5, 138.0, 142.3, 156.9, 162.2, 189.4

$243379,3234\left(\mathrm{NH}, \mathrm{NH}_{2}\right)$, 3057 (CH arom.), 2919, 2886 (CH aliph.), 1669 (C=O $1586(\mathrm{C}=\mathrm{N}), 1273(\mathrm{C}=\mathrm{S})$

$2.3\left(\mathrm{~s}, 3 \mathrm{H}, \mathrm{CH}_{3}\right), 4.5\left(\mathrm{~s}, 2 \mathrm{H}, \mathrm{NH}_{2}, \mathrm{D}_{2} \mathrm{O}\right.$-exchangeable), 6.9, $392\left[\mathrm{M}^{+}\right]$ 8.6 (2s, 2H, 2CH quinoline), 7.1-8.2 (m, 8H, Ar-H), $10.9 \quad$ (9.6), 127 , (s, $1 \mathrm{H}, \mathrm{NH}, \mathrm{D}_{2} \mathrm{O}$-exchangeable) 20.8, 81.7, 121.6, 122.9, 127.6, 18 (2), 128.3, 128.5, 128.8, 129.1 (2), 129.5, 133.5 (2), 138.2, 141.6, 153.3, 164.1, 189.6

$253367,3286\left(\mathrm{NH}_{1} \mathrm{NH}_{2}\right)$ 4.5 (s, 1H, $\mathrm{NH}_{2}, \mathrm{D}_{2} \mathrm{O}$-exchangeable), 7.1, $8.6\left(2 \mathrm{~s}, 2 \mathrm{H}, 2 \mathrm{CH} 396\left[\mathrm{M}^{+}\right]\right.$ 3100 (CH arom.), 1667 (C=O), quinoline), 7.4-8.1 (m, 8H, Ar-H), 9.7 (s, 1H, NH, $1589(\mathrm{C}=\mathrm{N}), 1224(\mathrm{C}=\mathrm{S}) \quad \mathrm{D}_{2} \mathrm{O}$-exchangeable)

(14.1), 82.0, 117.0 (2), 123.7, 126.9, 127.5, 127.7, 127.9 (2), 128.4, $131.4,131.5,132.7,144.0,145.7,152.9,160.9,164.8,185.9$

$263415,3339\left(\mathrm{NH}, \mathrm{NH}_{2}\right)$ 3071 (CH arom.), 2961, 2831 (CH aliph.), $1683(\mathrm{C}=\mathrm{O})$ $1586(\mathrm{C}=\mathrm{N}), 1234(\mathrm{C}=\mathrm{S})$ $3.8\left(\mathrm{~s}, 3 \mathrm{H}, \mathrm{OCH}_{3}\right), 4.5\left(\mathrm{~s}, 2 \mathrm{H}, \mathrm{NH}_{2}, \mathrm{D}_{2} \mathrm{O}\right.$-exchangeable), $408\left[\mathrm{M}^{+}\right]$ 7.1, 8.6 (2s, 2H, 2CH quinoline), 7.3-8.0 (m, 8H, Ar-H), 9.7 (8.7), 93 , (s, $1 \mathrm{H}, \mathrm{NH}, \mathrm{D}_{2} \mathrm{O}$-exchangeable) (100) $55.4,81.9,115.3$ (2), 123.5, 126.9, 127.2, 127.5, 127.7, 127.8 (2), $128.5,130.1,132.7,144.1,145.7,153.0,160.2,160.9,186.1$

27 3286, $3212\left(\mathrm{NH}, \mathrm{NH}_{2}\right)$, 3068 ( $\mathrm{CH}$ arom.), $1658(\mathrm{C}=\mathrm{O}), 1591(\mathrm{C}=\mathrm{N})$, $1202(\mathrm{C}=\mathrm{S})$ 4.5 (s, 2H, $\mathrm{NH}_{2}, \mathrm{D}_{2} \mathrm{O}$-exchangeable), 7.0, $8.6\left(2 \mathrm{~s}, 2 \mathrm{H}, 2 \mathrm{CH} 423\left[\mathrm{M}^{+}\right]\right.$ quinoline), 7.4-8.2 (m, 8H, Ar-H), 11.6 (s, 1H, NH, (44.9), $\mathrm{D}_{2} \mathrm{O}$-exchangeable) $102(100)$ 86.3, 121.0 (2), 122.8, 123.5, 124.5, 127.3, 128.7 (2), 130.5 (2), 135.6, 139.1, 139.6, 142.8, 144.0, 157.2, 165.7, 187.6 
28 3310, $3286\left(\mathrm{NH}_{1} \mathrm{NH}_{2}\right), \quad 4.5\left(\mathrm{~s}, 2 \mathrm{H}, \mathrm{NH}_{2}, \mathrm{D}_{2} \mathrm{O}\right.$-exchangeable), 7.0, $8.5\left(2 \mathrm{~s}, 2 \mathrm{H}, 2 \mathrm{CH} 457\left[\mathrm{M}^{+}\right]\right.$ 3100 (CH arom.), 1691 (C=O), quinoline), 7.2-8.2 (m, 8H, Ar-H), 11.1 (s, 1H, NH, $1589(\mathrm{C}=\mathrm{N}), 1261(\mathrm{C}=\mathrm{S}) \quad \mathrm{D}_{2} \mathrm{O}$-exchangeable)

91.2, 118.6, 123.5, 124.5, 125.5, 127.2, 128.2, $128.4(2), 130.4$,

(100) 130.5 (2), 131.4, 137.0, 139.8, 145.1, 156.1, 166.9, 189.6

$293229,3186\left(\mathrm{NH}_{1} \mathrm{NH}_{2}\right), \quad 4.5\left(\mathrm{~s}, 2 \mathrm{H}, \mathrm{NH}_{2}, \mathrm{D}_{2} \mathrm{O}\right.$-exchangeable), 7.0, $8.5\left(2 \mathrm{~s}, 2 \mathrm{H}, 2 \mathrm{CH} 504\left[\mathrm{M}^{+}\right]\right.$ 3081 (CH arom.), 1686 (C=O), quinoline), 7.1-8.2 (m, 8H, Ar-H), 11.1 (s, 1H, NH, (42.4), $1587(\mathrm{C}=\mathrm{N}), 1247(\mathrm{C}=\mathrm{S}) \quad \mathrm{D}_{2} \mathrm{O}$-exchangeable $) \quad 234(100)$ 88.7, 95.2, 125.7, 127.2, 127.5, 128.2, $128.6(2), 128.6,130.8$, $135.1,136.3,137.0(2), 138.0,147.6,155.1,162.9,189.0$

33 3406, $3381\left(\mathrm{NH}, \mathrm{NH}_{2}\right)$ 3051 (CH arom.), 2936, 2.3-3.0 (m, 6H, 3CH $\mathrm{CH}_{2}$ cyclo), 7.1, $8.6(2 \mathrm{~s}, 2 \mathrm{H}, 2 \mathrm{CH}$ $309\left[\mathrm{M}^{+}\right]$ 2847 ( $\mathrm{CH}$ aliph.), $1680(\mathrm{C}=\mathrm{O}), \mathrm{D}_{2} \mathrm{O}$-exchangeable) $1590(\mathrm{C}=\mathrm{N})$

22.6, 28.8, 37.7, 116.6, 125.1, 125.5 (2), 125.9, 126.4, 127.9, $128.3,135.1,140.8,141.6,144.5,165.0,165.9$

34 3247, $3176\left(\mathrm{NH}, \mathrm{NH}_{2}\right)$, 1.3-2.7 (m, 8H, 4CH 2 cyclo), 7.2, $8.6(2 \mathrm{~s}, 2 \mathrm{H}, 2 \mathrm{CH}$ 3091 (CH arom.), 2926, quinoline), 7.7-8.2 (m, 6H, $\left.\mathrm{Ar}-\mathrm{H}+\mathrm{NH}_{2}\right), 10.5(\mathrm{~s}, 1 \mathrm{H}, \mathrm{NH}$, 2853 (CH aliph.), 1657 (C=O), $\mathrm{D}_{2} \mathrm{O}$-exchangeable) $1610(\mathrm{C}=\mathrm{N})$ 22.0, 22.9, 24.0, 25.3, 110.2, 120.0, 126.7 (2), 127.7, 128.1, $128.5,129.6,132.2,136.8(2), 146.2,161.2,167.1$

35 3420, $3381\left(\mathrm{NH}, \mathrm{NH}_{2}\right)$, 3100 (CH arom.), 2927, 1.2-2.6 (m, 10H, 5 $\mathrm{CH}_{2}$ cyclo), 7.0, $8.6(2 \mathrm{~s}, 2 \mathrm{H}, 2 \mathrm{CH}$ quinoline), 7.4-7.9 (m, 6H, Ar- $\left.\mathrm{H}+\mathrm{NH}_{2}\right), 10.7(\mathrm{~s}, 1 \mathrm{H}, \mathrm{NH}$, 2861 ( $\mathrm{CH}$ aliph.), 1676 ( $\mathrm{C}=\mathrm{O}), \mathrm{D}_{2} \mathrm{O}$-exchangeable) $1599(\mathrm{C}=\mathrm{N})$ 
in an atmosphere of $5 \% \mathrm{CO}_{2}$. Forty-eight hours after drug addition, cells were fixed with $50 \%$ trichloroacetic acid at $4{ }^{\circ} \mathrm{C}(50 \mu \mathrm{L}$ per well) for $1 \mathrm{~h}$, washed with $1 \%$ acetic acid and stained for $30 \mathrm{~min}$ with $50 \mu \mathrm{L}$ of $0.4 \%(\mathrm{~m} / \mathrm{V})$ sulphorhodamine (SRB) dissolved in $1 \%$ ace-

Table III. In vitro cytotoxic activity of the newly synthesized compounds 1, 4-29, 33-35 against the human breast cancer cell line (MCF7)

\begin{tabular}{|c|c|c|}
\hline $\begin{array}{l}\text { Compound } \\
\text { No. }\end{array}$ & $\begin{array}{c}I C_{50} \\
\left(\mu \mathrm{mL}^{-1}\right)\end{array}$ & $\begin{array}{c}I C_{50} \\
\left(\mu \mathrm{mol} \mathrm{L}{ }^{-1}\right)\end{array}$ \\
\hline 1 & 22.7 & 107.5 \\
\hline 4 & 39.7 & 144.3 \\
\hline 5 & 19.1 & 62.2 \\
\hline 6 & 15.7 & 39.0 \\
\hline 7 & 15.4 & 37.0 \\
\hline 8 & 18.7 & 44.7 \\
\hline 9 & 24.4 & 56.4 \\
\hline 10 & 33.1 & 77.3 \\
\hline 11 & 18.5 & 42.3 \\
\hline 12 & 22.6 & 51.8 \\
\hline 13 & NA & NA \\
\hline 14 & NA & NA \\
\hline 15 & 23.9 & 53.4 \\
\hline 16 & NA & NA \\
\hline 17 & NA & NA \\
\hline 18 & 43.7 & 90.4 \\
\hline 19 & NA & NA \\
\hline 20 & 40.1 & 98.2 \\
\hline 21 & 10.7 & 40.2 \\
\hline 22 & NA & NA \\
\hline 23 & NA & NA \\
\hline 24 & 22.4 & 57.1 \\
\hline 25 & 11.8 & 29.7 \\
\hline 26 & NA & NA \\
\hline 27 & 11.3 & 26.7 \\
\hline 28 & 24.4 & 53.3 \\
\hline 29 & NA & NA \\
\hline 33 & 20.5 & 66.3 \\
\hline 34 & 10.7 & 33.1 \\
\hline 35 & 12.7 & 37.6 \\
\hline Doxorubicin & 26.3 & 47.9 \\
\hline
\end{tabular}

NA - no activity observed under experimental conditions. 
tic acid. Excess unbound dye was removed by four washes with $1 \%$ acetic acid and the attached stain was recovered with Tris-EDTA buffer. Colour intensity was measured using an enzyme-linked immunosorbent assay ELISA reader (BMG LABTECH GmbH, Germany). Absorbance was measured at $510 \mathrm{~nm}$. The relation between the surviving fraction and drug concentration was plotted to get the survival curve for the breast cancer cell line (MCF7) after specified time (29). The molar concentration required for $5 \%$ inhibition of cell viability $\left(I C_{50}\right)$ was preliminarily calculated from the constructed dose-response curve using the Prism software (Graphpad, Inc., La Jolla, USA) and the results are presented in Table III.

\section{RESULTS AND DISSCUSION}

\section{Chemistry}

Schemes 1 and 2 outline the synthetic pathways used to obtain compounds $\mathbf{1 - 3 5}$. The solvent-free reaction of aryl amines with ethyl cyanoacetate is well known as one of the most widely used synthetic methods. Thermal fusion of 3-aminoquinoline with ethyl cyanoacetate at $220^{\circ} \mathrm{C}$ afforded the corresponding 2-cyano- $N$-(quinolin- $N$-3-yl)acetamide (1). The structure of novel compounds was established on the basis of elemental analysis and spectral data. IR spectrum of compound 1 showed bands at $3260 \mathrm{~cm}^{-1} \mathrm{NH}$, and $2201 \mathrm{~cm}^{-1}$ $\mathrm{C} \equiv \mathrm{N}$, while ${ }^{1} \mathrm{H}-\mathrm{NMR}$ spectrum revealed a singlet at $4.0 \mathrm{ppm}$ assigned to the $\mathrm{CH}_{2}$ group. In addition, the structure of compound $\mathbf{1}$ was confirmed through X-ray crystallography (30) (Figs. 2 and 3).

The present study was aimed to prepare novel quinolone derivatives using cyanoacetamide 1 as strategic starting material. Thus, interaction of compound 1 with acetylacetone furnished the corresponding 4,6-dimethyl-2-oxo-1-(quinolin-3-yl)-1,2-dihydropyridine-3-carbonitrile 4 . It can be postulated that the reaction initially proceeded via a nucleophilic attack to form Michael adduct 2 which in turn cyclized to product 3 . Elimination of two molecules of water gave 1,2-dihydropyridine 4 . IR spectrum of 4 showed the absence of $\mathrm{NH}_{2}$ group and the presence of bands at 2937 and $2870 \mathrm{~cm}^{-1}\left(\mathrm{CH}\right.$ aliphatic), $2218 \mathrm{~cm}^{-1}$ $(\mathrm{C} \equiv \mathrm{N})$, and $1658 \mathrm{~cm}^{-1}(\mathrm{C}=\mathrm{O})$. Its ${ }^{1} \mathrm{H}$ NMR spectrum displayed the following signals: $2.0 \mathrm{ppm}$ for $2 \mathrm{CH}_{3}$ and $6.5 \mathrm{ppm}$ for pyridinone $\mathrm{CH}$. Interaction of 4 with elemental sulfur in ethanolic triethylamine furnished the corresponding thienopyridine derivative 5. Its IR spectrum revealed characteristic bands at 3406, $3326\left(\mathrm{NH}_{2}\right), 1657 \mathrm{~cm}^{-1}(\mathrm{C}=\mathrm{O})$, and $1611 \mathrm{~cm}^{-1}(\mathrm{C}=\mathrm{N}) .{ }^{1} \mathrm{H}$
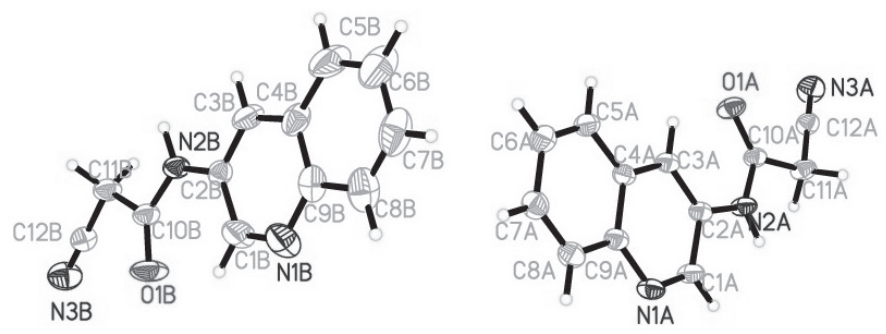

Fig. 2. Oak Ridge thermal ellipsoid plot (ORTEP) diagram of the title compound 1 drawn at $40 \%$ ellipsoids for non-hydrogen atoms. 


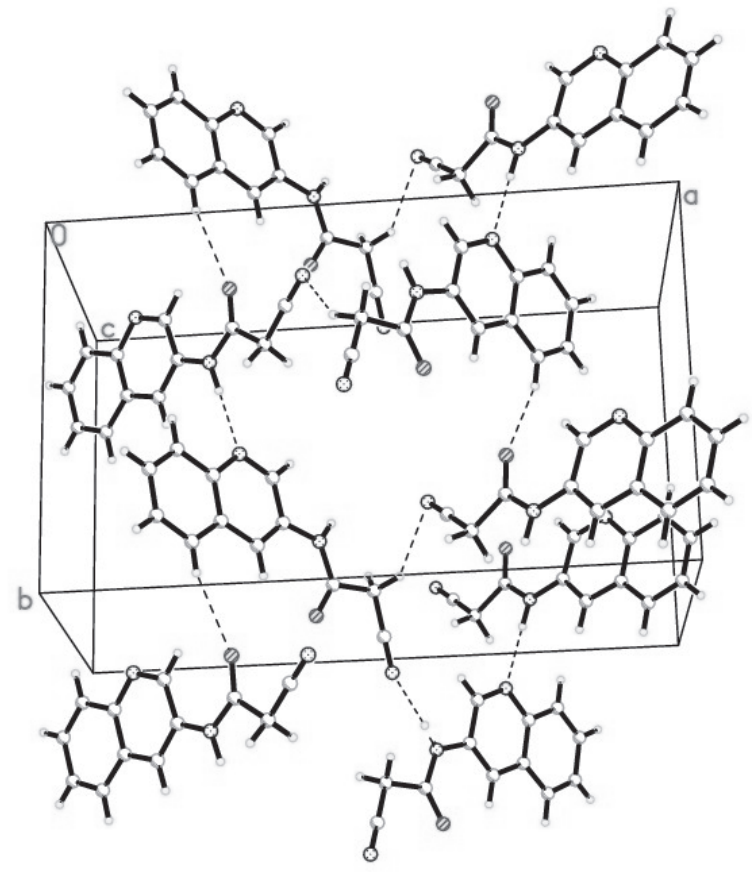

Fig. 3. Crystal packing of compound 1 showing intermolecular hydrogen bonds as dashed lines.

NMR spectrum showed signals at $1.6 \mathrm{ppm}$ for $\mathrm{CH}_{3}, 5.1 \mathrm{ppm}$ for pyridinone $\mathrm{CH}$, and $6.6 \mathrm{ppm}$ for thiophene $\mathrm{CH} .{ }^{13} \mathrm{C}$ NMR spectrum revealed a singlet at $161.2 \mathrm{ppm}$ assigned to $\mathrm{C}=\mathrm{O}$. Application of Michael addition of 4 to benzylidinemalononitriles in absolute ethanol in the presence of piperidine yielded the corresponding 1,2-dihydroisoquinoline derivatives 6-20, via loss the of $\mathrm{HCN}$ molecule (Scheme 1). IR spectra of compounds 6-20 revealed the presence of bands for $\mathrm{NH}_{2}, \mathrm{C} \equiv \mathrm{N}, \mathrm{C}=\mathrm{O}$ and $\mathrm{C}=\mathrm{N}$. ${ }^{1} \mathrm{H}$ NMR spectra of compounds 6-20 exhibited a singlet at 6.2-4.4 ppm assigned to the $\mathrm{NH}_{2}$ group. Treatment of 1 with dimethylformamidedimethylacetal (DMF-DMA) in dry xylene gave the corresponding enamine derivative 21 . IR spectrum showed bands at $3287 \mathrm{~cm}^{-1}(\mathrm{NH}), 2224 \mathrm{~cm}^{-1}(\mathrm{C} \equiv \mathrm{N})$, and $1688 \mathrm{~cm}^{-1}(\mathrm{C}=\mathrm{O}) .{ }^{1} \mathrm{H}$ NMR spectrum revealed signals at $2.6 \mathrm{ppm}$ for $\mathrm{N}\left(\mathrm{CH}_{3}\right), 7.5 \mathrm{ppm}$ for the $\mathrm{CH}$ group and 8.9 ppm for the $\mathrm{NH}$ group. Interaction of compound 1 with 2-sulfanylacetic acid in pyridine produced the corresponding thiazolidine derivative 22. Compound $\mathbf{2 2}$ was characterized by the presence of a strong absorption band at $1705 \mathrm{~cm}^{-1}$ in the IR spectrum specific for the thiazolidinone as a proof of cyclization. In the ${ }^{1} \mathrm{H}$ NMR spectrum, it was the presence of a singlet equivalent to two protons at $3.7 \mathrm{ppm}$, representing the C-5 protons of the thiazolidinone nucleus. The ${ }^{13} \mathrm{C}$ NMR spectrum of compound 22 showed signals at 174.1 and $32.1 \mathrm{ppm}$ (C-4 and C-5 in the thiazolidinone nucleus). On the other hand, 4-amino-3substituted-N-(quinolin-3-yl)-2-thiox-2,3-dihydrothiazde-5-carboxamides 23-29 were obtained by the reaction of $\mathbf{1}$ with sulfur and aryl isothiocyanate in DMF-EtOH containing triethylamine as a catalyst. IR spectra of compounds 23-29 showed bands for the $\mathrm{NH}, \mathrm{NH}_{2}$, $\mathrm{C}=\mathrm{O}, \mathrm{C}=\mathrm{N}, \mathrm{C}=\mathrm{S}$ groups. ${ }^{1} \mathrm{H}$ NMR spectra revealed a singlet at $4.5 \mathrm{ppm}$ assigned to the $\mathrm{NH}_{2}$ 
group. ${ }^{13} \mathrm{C}$ NMR spectra of compounds $23-29$ revealed signals at $189.6-183.9 \mathrm{ppm}$ for $\mathrm{C}=\mathrm{S}$ groups.

Finally, the corresponding thiophene derivatives 33-35 were obtained via the reaction of 1 with cyclopentanone or cyclohexanone and/or cycloheptanone and sulfur in a mixture of $\mathrm{DMF} / \mathrm{EtOH}$ containing a catalytic amount of morpholine. The reaction may be explained via the intermediates 30-32 (Scheme 2). Their IR spectra revealed bands at 3420-3176 cm-1 $\left(\mathrm{NH}, \mathrm{NH}_{2}\right)$ and $1680-1657 \mathrm{~cm}^{-1}(\mathrm{C}=\mathrm{O}) .{ }^{1} \mathrm{H}$ NMR spectrum of 33 exhibited signals at 2.3-3.0 ppm for the 3-cyclopentyl $\mathrm{CH}_{2}$ group. ${ }^{1} \mathrm{H}$ NMR spectrum of 34 showed signals at 1.3-2.7 ppm for the 4-cyclohexyl $\mathrm{CH}_{2}$ group. ${ }^{1} \mathrm{H}$ NMR spectrum of 35 revealed signals at 1.2-2.6 ppm corresponding to the 5-cycloheptyl $\mathrm{CH}_{2}$ group.

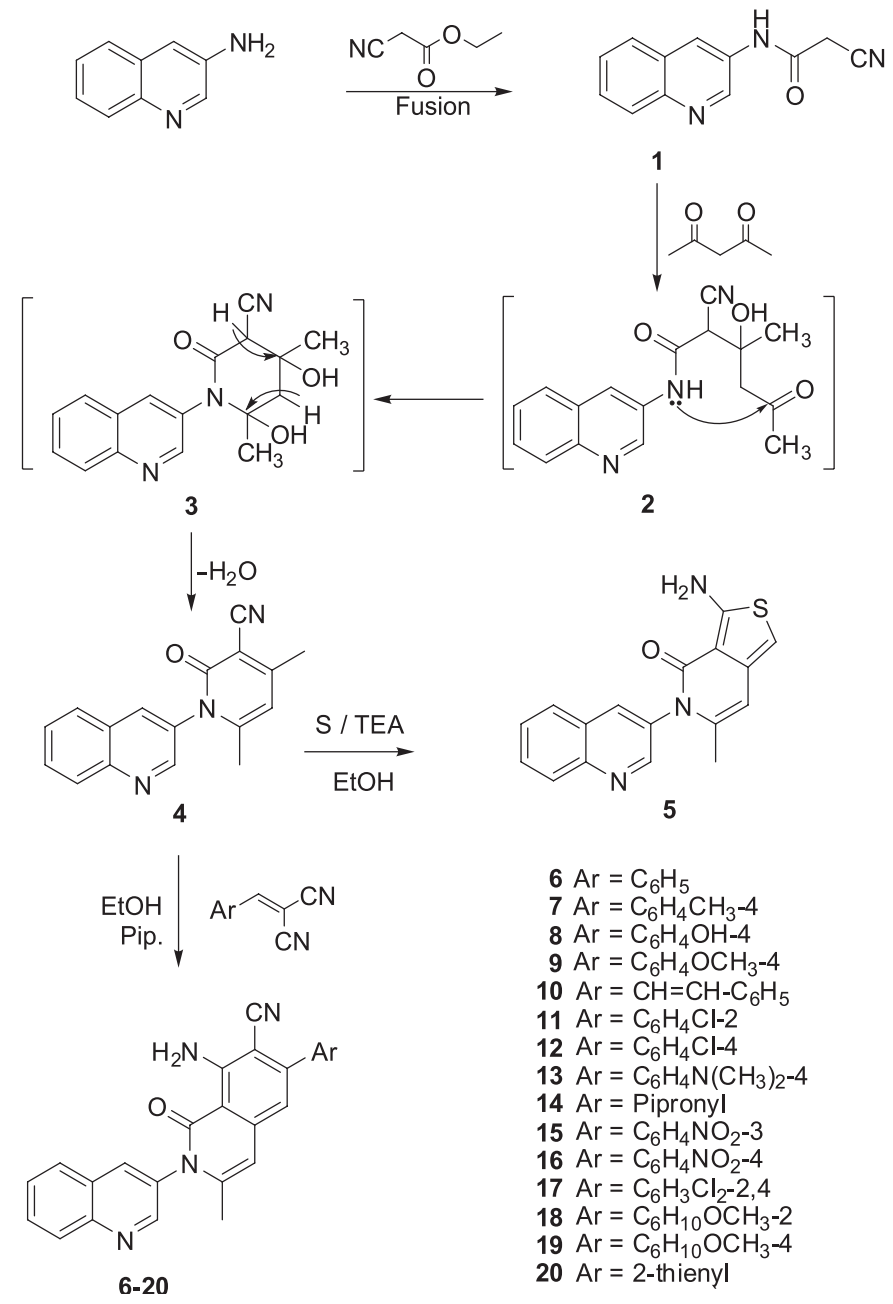

Schemes 1 


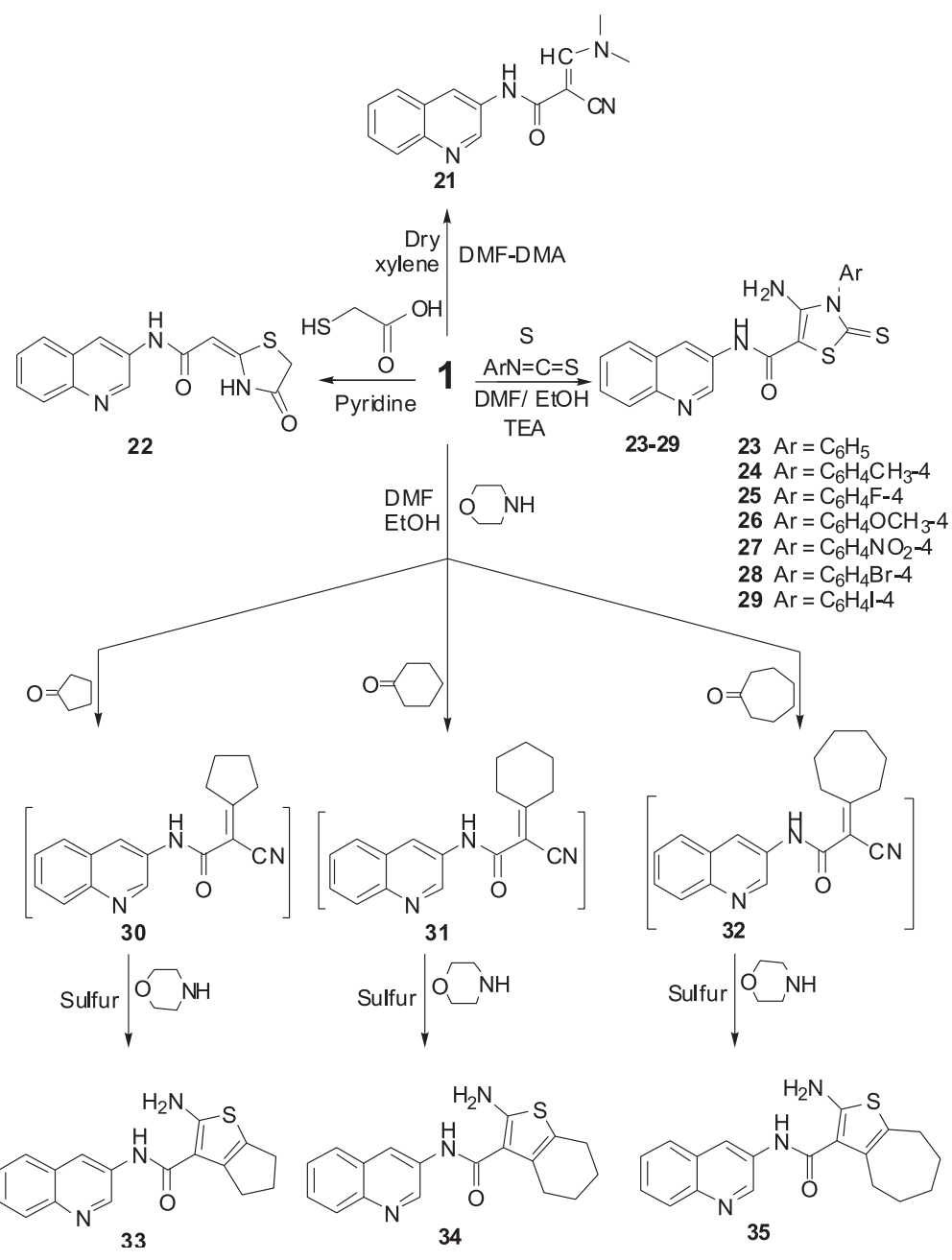

Schemes 2

\section{In vitro antitumor activity}

The $I C_{50}$ concentrations are presented in Table III. Most of the synthesized compounds showed better cytotoxic activity than doxorubicin $\left(I C_{50} 47.9 \mu \mathrm{mol} \mathrm{L}^{-1}\right)$ against the MCF7 cell line except compounds 1, 4, 18 and 20 (IC $C_{50}$ values: $\left.98-108 \mu \mathrm{mol} \mathrm{L}^{-1}\right)$. The quinoline derivative carrying dihydrothiazole with 4-nitrophenyl and thioxo moiety (27), dihydrothiazole with 4-fluorophenyl and thioxo moiety (25), tetrahydrobenzothiophene (34), dihydroisoquinoline with 4-methylphenyl and carbonitrile moiety (7), tetrahydro-4-H-cycloheptathiophene (35), dihydroisoquinoline with unsubstituted phenyl moiety (6), acrylamide moiety (21), dihydroisoquinoline with 2-chlorophenyl moiety (11), dihydroisoquinoline 
with 4-hydroxyphenyl moiety (8) with exhibited good cytotoxic activity better than doxorubicin as reference drug $\left(I C_{50} 26.7\right.$ to $\left.44.7 \mu \mathrm{mol} \mathrm{L}^{-1}\right)$. A closer look into the structure activity relationship indicates that addition of dihydrothiazole with thioxo group at 2-position, 4-nitrophenyl at 3-position, free amino group at 4-position, dihydrothiazole with thioxo group at 2-position, 4-fluorophenyl at 3-position, free amino group at 4-position and tetrahydrobenzo[b]thiophene with free amino group at 2-position was proven to be successful in the case of compounds 27, 25 and 34, which showed an increase in activity. Also, quinolone incorporating tetrahydrobenzo[b]thiophene (34) with $I C_{50} 33.1 \mu \mathrm{mol} \mathrm{L}{ }^{-1}$ exhibited higher activity than cyclopenta[b]thiophene (33) with $I C_{50} 66.3 \mu \mathrm{mol} \mathrm{L}-1$. The presence of the cyclohepta[b]thiophene ring in compound (35) $\left(I C_{50} 37.6 \mu \mathrm{mol} \mathrm{L}-1\right)$ enhanced the anti-breast cancer activity more than the corresponding cyclopenta[b]thiophene (33) $\left(I C_{50}\right.$ $66.3 \mu \mathrm{mol} \mathrm{L}{ }^{-1}$ ). In addition, quinoline bearing dihydroisoquinoline with 4-chlorophenyl (12), dihydroisoquinoline with 3-nitrophenyl moiety (15) and dihydrothiazole with 4-bromophenyl moiety (28) were nearly as active as doxorubicin $\left(I C_{50} 51.8\right.$ to $\left.53.4 \mu \mathrm{mol} \mathrm{L}^{-1}\right)$. On the other hand, compounds 5, 9, 10, 24 and 33 exhibited moderate activity. Compounds $\mathbf{1}$, 4, 18 and 20 were the least active compounds in this series compared to doxorubicin, while the remaining compounds revealed no activity.

\section{CONCLUSIONS}

The objective of the present study was to synthesize and investigate the anti-breast cancer activity of some novel quinoline derivatives bearing biologically active moieties. All the synthesized compounds were characterized and evaluated for their anti-breast cancer activity and it was found that 4-amino-3-(4-nitrophenyl)- $N$-(quinolin-3-yl)-2-thioxo-2,3-dihydrothiazole-5-carboxamide (27), 4-amino-3-(4-fluorophenyl)- $N$-(quinolin-3-yl)-2-thioxo-2, 3-dihydrothiazole-5-carboxamide (25), 2-amino- $N$-(quinolin-3-yl)-4,5,6,7-tetrahydrobenzo[b] thiophene-3-carboxamide (34), 8-amino-3-methyl-1-oxo-2-(quinolin-3-yl)-6- $p$-tolyl-1,2dihydroisoquinoline-7-carbonitrile (7), 2-amino- $\mathrm{N}$-(quinolin-3-yl)-5,6,7,8-tetrahydro- $4 \mathrm{H}$ cyclohepta[b] thiophene-3-carboxamides (35), 8-amino-3-methyl-1-oxo-6-phenyl-2-(quinolin-3-yl)-1,2-dihydroisoquinoline-7-carbonitrile (6), 2-cyano-3-(dimethylamino)- $N$ (quinolin-3-yl)acrylamide (21), 8-amino-6-(2-chlorophenyl)-3-methyl-1-oxo-2-(quinolin-3yl)-1,2-dihydroisoquinoline-7-carbonitrile (11) and 8-amino-6-(4-hydroxyphenyl)-3-methyl1-oxo-2-(quinolin-3-yl)-1,2-dihydroisoquinoline-7-carbonitrile (8) exhibited anti-breast cancer activity higher than the positive control doxorubicin. Also, it was found that 8-amino-6-(4-chlorophenyl)-3-methyl-1-oxo-2-(quinolin-3-yl)-1,2-dihydroisoquinoline-7carbonitrile (12), 4-amino-3-(4-bromophenyl)- $N$-(quinolin-3-yl)-2-thioxo-2,3-dihydrothiazole-5-carboxamide (28), and 8-amino-3-methyl-6-(3-nitrophenyl)-1-oxo-2-(quinolin-3-yl)-1, 2-dihydroisoquinoline-7-carbonitrile (15) are almost as active as doxorubicin.

Acknowledgements. - The authors extend their sincere appreciation to the Deanship of Scientific Research at King Saud University for its funding of this research through the Research Group Project No. RGP-VPP- 302.

\section{REFERENCES}

1. H. Frankish, 15 Million new cancer cases per year by 2020, says WHO, Lancet 361 (2003) 1278-1287; DOI: 10.1016/S0140-6736(03)13038-3. 
2. K. Kubo, T. Shimizu, S. I. Ohyama, H. Murooka, A. Iwai, K. Nakamura, K. Hasegawa, Y. Kobayashi, N. Takahashi, K. Takahashi, S. Kato, T. Izawa and T. Isoe, Novel potent orally active selective VEGFR-2 tyrosine kinase inhibitors: synthesis, structure-activity relationships, and antitumor activities of $N$-phenyl-N'-\{4-(4-quinolyloxy)phenyl\}-ureas, J. Med. Chem. 48 (2005) 1359-1366; DOI: $10.1021 / \mathrm{jm} 030427 \mathrm{r}$.

3. R. O'Connor, Mdm-mediated ubiquitylation: P53 and beyond, a review of mechanisms of circumvention and modulation of chemotherapeutic drug resistance, Curr. Cancer Drug Targets 9 (2009) 273-280; DOI: 10.2174/156800909788166583.

4. C. Hu, V. R Solomon and G. L. Ulibarri, The efficacy and selectivity of tumor cell killing by AKT inhibitors are substantially increased by chloroquin, Bioorg. Med. Chem. 16 (2008) 7888-7893; DOI: 10.1016/j.bmc.2008.07.076.

5. Y. C. Mayur, G. J. Peters, V. V. Prasad, C. Lemo and N. K .Sathish, Design of new drug molecules to be used in reversing multidrug resistance in cancer, Curr. Cancer Drug Targets 9 (2009) 298-306; DOI: $10.2174 / 156800909788166619$.

6. M. G. Ferlin, B. Gatto, G. Chiarelotto and M. Palumbo, Pyrroloquinoline derivatives as potential antineoplastic drugs, Bioorg. Med. Chem. 8 (2000) 1415-1422; DOI: 10.1016/S0968-0896(00)00060-2.

7. M. S. Alsaid, M. S. Bashandy, S. I. Al-Qasoumi and M. M. Ghorab, Anti-breast cancer activity of some novel 1,2-dihydropyridine, thiophene and thiazole derivatives, Eur. J. Med. Chem. 46 (2011) 137-141; DOI: 10.1016/j.ejmech.2010.10.024.

8. S. Pédeboscq, D. Gravier, F. Casadebaig, G. Hou, A. Gissot, F. De. Giorgi, F. Ichas, J. Cambar and J. P. Pometan, Synthesis and study of antiproliferative activity of novel thienopyrimidines on glioblastoma cells, Eur. J. Med. Chem. 45 (2010) 2473-2479; DOI:10.1016/j.ejmech.-2010.02.032.

9. R. L. Weinkauf, A. Y. Chen, C. Yu, L. Liu, L. Barrows and E. J. LaVoie, Antineoplastic activity of benzimidazo[1,2-b] isoquinolines, indolo[2,3-b]quinolines, and pyridocarbazoles, Bioorg. Med. Chem. 2 (1994) 781-786; DOI: 10.1016/S0968-0896(00)82177-X.

10. S. Li, Z. Yao, Y. Zhao, W. Chen, H. Wang, X. Kuang, W. Zhan, S. Yao, S. Yu and W. Hu, Design, synthesis and biological evaluation of novel acrylamide analogues as inhibitors of BCR-ABL kinase, Bioorg. Med. Chem. Lett. 22 (2012) 5279-5282; DOI: 10.1016/j.bmcl.2012.06.044.

11. F. E. Onen-Bayram, I. Durmaz, D. Scherman, J. Herscovici and R. Cetin-Atalay, A novel thiazolidine compound induces caspase-9 dependent apoptosis in cancer cells, Bioorg. Med. Chem. 20 (2012) 5094-5102; DOI: 10.1016/j.bmc.2012.07.016.

12. G. J. Atwell, C. D. Bos, B. C. Baguley and W. A. Denny, Potential antitumor agents. 56. "Minimal" DNA-intercalating ligands as antitumor drugs: phenylquinoline-8-carboxamides, J. Med. Chem. 3 (1988) 1048-1052; DOI: 10.1021/jm00400a029.

13. G. J. Atwell, B. C. Baguley and W. A. Denny, Potential antitumor agents. 57. 2-phenylquinoline-8carboxamides as minimal DNA-intercalating antitumor agents with in vivo solid tumor activity, J. Med. Chem. 32 (1989) 396-401; DOI: 10.1021/jm00122a018.

14. T. Suzuki, N. Fukazawa, K. San-nohe, W. Sato, O. Yano and T. Tsuruo, Structure activity relationship of newly synthesized quinoline derivatives for reversal of multidrug resistance in cancer, J. Med. Chem. 40 (1997) 2047-2052; DOI: 10.1021/jm9608691.

15. Y. H. Wang, T. Motoji, S. Motomura, N. Shiozaki, T. Tsuruo and H. Mizoguchi, Multi-drug resistance-reversing agent, on acute myelogenous leukaemic blasts and K562 cells resistant to adriamycin cell line, Eur. J. Haematol. 58 (1997) 186-194; DOI: 10.1111/j.1600-0609.1997.tb00946.x.

16. H. Zhao, Y. Cai, S. Santi, R. Lafrenie and H. Lee, Chloroquine-mediated radiosensitization is due to the destabilization of the lysosomal membrane and subsequent induction of cell death by necrosis, Radiat. Res. 164 (2005) 250-257; DOI: 10.1667/RR3436.1. 
17. H. Zhang, V. R. Solomon, C. Hu, G. Ulibarri and H. Lee, Synthesis and in vitro cytotoxicity evaluation of 4-aminoquinoline derivatives, Biomed. Pharmacother. 62 (2008) 65-69; DOI: 10.1016/j.biopha.2007.04.007.

18. V. R. Solomon and H. Lee, Chloroquine and its analogs: a new promise of an old drug for effective and safe cancer therapies, Eur. J. Pharmacol. 625 (2009) 220-233; DOI: 10.1016/j.ejphar.2009.06.063.

19. M. S. Al-Said, M. G. El-Gazzar, M. S. Al-Dosari and M. M. Ghorab, Synthesis, anticancer activity and radiosensitizing evaluation of some new 2-pyridone derivatives, Arzneimittel-Forsch. 62 (2012) 149-156; DOI: 10.1055/s-0031-1299695.

20. P. Fossa, G. Menozzi, P. Dorigo, M. Floreani and L. Mosti, Synthesis and pharmacological characterization of functionalized 2-pyridones structurally related to the cardiotonic agent milrinone, Bioorg. Med. Chem. 11 (2003) 4749-4759; DOI: 10.1016/S0968-0896(03)00528-5.

21. W. Chen, P. Zhan, D. Rai, E. De Clercq, C. Pannecouque, J. Balzarini, Z. Zhou, H. Liu and X. Liu, Discovery of 2-pyridone derivatives as potent HIV-1 NNRTIs using molecular hybridization based on crystallographic overlays, Bioorg. Med. Chem. 22 (2014) 1863-1872; DOI: 10.1016/j.bmc.2014.01.054.

22. M. M. Ghorab, Z. H. Ismail, M. Abdalla and A. A. Radwan, Synthesis, antimicrobial evaluation and molecular modelling of novel sulfonamides carrying a biologically active quinazoline nucleus, Arch. Pharm. Res. 36 (2013) 660-670; DOI: 10.1007/s12272-013-0094-6.

23. M. S. Al-Dosari, M. M. Ghorab, M. S. Alsaid and M. Y. Nissan, Discovering some novel 7-chloroquinolines carrying a biologically active benzenesulfonamide moiety as a new class of anticancer agents, Chem. Pharm. Bull. 61 (2013) 50-58; DOI: 10.1248/cpb.c12-00812.

24. M. S. Alsaid, M. S. Bashandy and M. M. Ghorab, Novel quinolines bearing a biologically active trimethoxyphenyl moiety as a new class of antitumor agents, Arzneimittel-Forsch. 61 (2011) 527531; DOI: 10.1055/s-0031-1296239.

25. M. S. Bashandy, M. S. Al-Said, S. I. Al-Qasoumi and M. M. Ghorab, Design and synthesis of some novel hydrazide, 1, 2-dihydropyridine, chromene derivatives carrying biologically active sulfone moieties with potential anticancer activity, Arzneimittel-Forsch. 61 (2011) 521-526; DOI: 10.1055/s0031-1296238.

26. M. M. Ghorab, M. S. Al-Said and E. M. El-Hossary, In vitro cytotoxic evaluation of some new heterocyclic sulfonamide derivatives, J. Heterocycl. Chem. 48 (2011) 563-571; DOI: 10.1002/jhet.619.

27. M. S. Al-Said, M. S. Bashandy, S. I. Al-Qasoumi and M. M. Ghorab, Anti-breast cancer activity of some novel 1,2-dihydropyridine, thiophene and thiazole derivatives, Eur. J. Med. Chem. 46 (2011) 137-141; DOI: 10.1016/j.ejm-ech.2010.10.024.

28. M. M. Ghorab, F. A. Ragab, H. I. Heiba, R. K. Arafa and E. M. El-Hossary, Docking study, in vitro anticancer screening and radiosensitizing evaluation of some new fluorine-containing quinoline and pyrimidoquinoline derivatives bearing a sulfonamide moiety, Med. Chem. Res. 20 (2011) 388400; DOI: 10.1007/s00044-010-9332-3.

29. P. Skehan, R. Storeng, D. Scudiero, A. Monks, J. McMahon, D. Vistica, J. T. Warren, H. Bokesch, S. Kenny and M. R. Boyd, New colorimetric cytotoxicity assay for anticancer-drug screening, J. Natl. Cancer. Inst. 82 (1990) 1107-1112; DOI: 10.1093/jnci/82.13.1107.

30. M. M. Ghorab, M. S. Alsaid, H. A. Ghabour and H.-K. Fun, Synthesis, crystal structure and antitumor activity of novel 2-cyano-N-(quinolin-3-yl) acetamide, Asian J. Chem. 26 (2014) 7389-7392; DOI:10.14233/ajchem.2014.17050. 